\title{
Neuroimaging in Psychiatry and Neurology: Current Status and Future Directions
}

\author{
Rajendra D. Badgaiyan ${ }^{1^{*}}$ and Kenneth Blum ${ }^{2}$ \\ ${ }^{1}$ Professor of Psychiatry and Director of Functional and Molecular Imaging, University of Minnesota, Minneapolis, MN, USA \\ ${ }^{2}$ Professor of Psychiatry at Keck School of Medicine, University of Southern California, Los Angeles, CA, USA
}

\section{Correspondence to:}

Rajendra D. Badgaiyan

Professor of Psychiatry and Director of

Functional and Molecular Imaging

University of Minnesota, Minneapolis, USA

E-mail: badgaiyan@gmail.com

Received: January 04, 2016

Accepted: January 07, 2016

Published: January 07, 2016

Citation: Badgaiyan RD, Blum K. 2016. Neuroimaging in Psychiatry and Neurology: Current Status and Future Directions. $J$ Neuroimaging Psychiatry Neurol 1(1): 1-3.

Copyright: (C) 2016 Badgaiyan and Blum. This is an Open Access article distributed under the terms of the Creative Commons Attribution 4.0 International License (CC-BY) (http:// creativecommons.org/licenses/by/4.0/) which permits commercial use, including reproduction, adaptation, and distribution of the article provided the original author and source are credited.

Published by United Scientific Group

\begin{abstract}
Modern neuroimaging techniques have made a significant contribution to our understanding of human brain and its regulatory mechanisms. These techniques however are limited by their ability to detect only a few dimensions of the brain physiology. There is therefore a need to expand the scope of neuroimaging to allow detection of all known dimensions of the brain activity. There is also a need to explore newer dimensions that affect and regulate brain function. Lastly, the focus of studied on the brain disorders is often directed to symptoms or syndromes which prevents us from understanding real neuropathology. There is therefore a need to change the approach and study brain disorders using neuroscientific techniques, psychological concepts, and philosophical vision.
\end{abstract}

\section{Editorial}

Rapid advancement in neuroimaging techniques in recent years has presented us with an incredible opportunity to study structure and function of live human brain in almost real time. Using these techniques, we can now visualize fine details of the brain anatomy, track neuronal connectivity and measure task-induced changes in hemodynamics, electric potentials and neurotransmitter concentration. These techniques have empowered us to study not only the structure but also different aspects of the brain function. This empowerment gives us hope that finally we will be able to understand complexities of the nervous system. In the past two decades neuroimaging data have helped us make significant advances in this direction. With these advances we now have better insight about disorders of the nervous system. We are however far from understanding many of these disorders, particularly those that present with psychiatric or psychosomatic symptoms.

One of the reasons for relative poor understanding of these conditions is our tendency of compartmentalizing knowledge. Because of the compartmentalization, conditions that have multidisciplinary dimension tend to get neglected or poorly addressed. Psychiatric and neuropsychiatric conditions are good examples. Since historically cognition, behavior, emotion and conscious experiences were considered psychological processes, these functions were not included in neuroscientific studies for a long time. Fortunately, neuroscientists now consider these processes as an integral part of the brain function. This change in perspective would surely help us better understand disorders of cognition, emotion, behavior and consciousness. Scientific inquiry on these disorders could use many strategies and tools. Neuroimaging is playing a major role in this context and in the foreseeable future it will remain the primary tool to study these processes. 
Studies on these processes require investigators to have familiarity not only with the brain sciences but also with psychological processes and philosophical concepts. Such a familiarity was not possible in the past because traditional compartmentalized teaching and research did not allow interdisciplinary cross talk. Modern neuroscience teaching and research however has recognized the importance of cross disciplinary expertise and research. As a result, we now have programs that allow interdisciplinary learning and training. Because of this change in perspective, investigators have begun to understand how cognitive, behavioral and emotional functions are processed in the brain. Based on this understanding we now have a better perspective on these processes and soon begin applying these findings in clinics to help patients with disorders of cognition, emotion and behavior. If this happens, a lot of uncertainty in treatment of psychiatric and neuropsychiatric conditions should go away and we will be more confident of treatment outcome.

But before that becomes a reality, we have to have a better understanding of the brain mechanisms that control and process our cognition, behavior and emotion. This understanding would require study of different aspects of the brain control mechanisms. It would include deeper understanding of known mechanisms of electrical and chemical signal transmission and exploration of the mechanisms that are understudied like biophotons, and mechanisms that have not yet been discovered.

There should also be a shift in the approach. Current approach of studying symptoms and syndromes limits our knowledge to the expression of deficit. There is therefore a need to shift the approach and begin exploring neurophysiological deficit rather than its manifestation. This approach would provide better understanding of the diseases of brain. It would however need us to look at the disease as a comprehensive deficit and not bind it to artificial compartments. Thus, understanding of memory deficits and processing would not only allow us to understand dementias but also a number of other neurological, psychiatric and metabolic conditions in which memory processing is impaired. Fortunately, there is a trend towards shift in approach in this direction. This trend needs to be encouraged and an academic environment that promotes this trend should be created.

This new approach would allow us to explore the basic mechanisms of brain and just symptoms. There are a number of brain mechanisms that are extremely important but rarely studied because of the lack of a directly identifiable disorder associated with it. One such mechanism is the initiation and execution of willed actions. At present we do not know how the brain makes a decision to initiate an action and how it activates the right neurons or group of neurons in the brain to execute the action. Making a willed action is one of the fundamental properties of a living being, but we have absolutely no clue how this action is executed. A breakthrough in understanding of this mechanism would go a long way in making us understand the basis of pathological actions and thought processes.

Neuroimaging obviously would play an important role in helping us answer these questions. There is however a need to expand the scope of neuroimaging. Currently these techniques can study only limited aspects of the brain function like changes in the structure, hemodynamic response, electrical potentials and neurotransmitter concentration. Study of these changes will definitely help us advance the knowledge of the brain and its functions but would not be adequate to make us completely understand the brain processing. We need additional techniques that can quantify other changes known to influence the brain activity. For example, the ability of dendrites to form synapses or the patterns of early migration of neurons and so on. In addition to the changes that are known we need to explore other changes that have not yet been discovered. Possibly through the advent of photogenetics some of these questions could be addressed in the future.

Thus, currently we are at an important cross road and our actions and approaches would define success of scientific understanding of diseases of the nervous system. There is a need to initiate debate on this issue and follow the approach that is most likely to succeed in years to come.

The Journal of Neuroimaging in Psychiatry and Neurology (JNPN) will provide a platform to advance the debate on current and future research strategies. It is also intended to be a forum for rapid communication of data. We propose to make the journal a platform for exchange of ideas and data on all aspects of neuroimaging that are relevant directly or indirectly to psychiatric or neurological disorders. Since other neuroimaging journals tend to make an artificial distinction between psychiatric and neurological conditions and expect to limit the discussion to a particular area, many investigators find it too restrictive. The JNPN will not make such a distinction and will encourage cross disciplinary perspective. In that respect this journal will be different from other existing journals. It will be different also in the speed of publication. On request of authors we will review and publish manuscript that have high potential impact within 10 days of receipt. Additionally, the journal will include papers that have significant negative results as well as manuscripts that provide hypotheses or future perspectives based on sound evidence. The manuscript will be accepted under the following categories:

1. Original Report: Original neuroimaging data relevant to any discipline including but not limited to psychiatry, neurology, cognition, behavior and emotion.

2. Review and Mini-review: Should be relevant to neuroimaging. Must provide novel perspective.

3. Negative Results: Neuroimaging data that did not validate the hypothesis examined but are still important either for making a concept or avoiding duplication of experiments will be published on experimental basis provided the significance of negative data is clearly discussed.

4. Perspective: This category is intended for theoretical papers that provide futuristic view of concepts or technologies. Authors will be allowed to use their imagination in these manuscripts.

5. Hypothesis: Hypotheses developed in these manuscripts are expected to be based on existing data. 
6. Letters to the Editor: Comments on the papers published in the journal in last 6 months will be included in this category.

7. Computational Neuroimaging: This category will include papers with original modeling and reviews or perspectives on modeling of neuroimaging data.

8. News and Discussion: This section will include news and discussion on technologies, concepts and significant papers published elsewhere.

9. Rapid Publication: Authors submitting manuscripts under any of the categories (except Review/Mini review) can request rapid publication if early dissemination of information is important. If adequate justification is provided, these manuscripts will be reviewed and published within 10 days of initial submission.

10. Preliminary Data: Data of extreme interest may be published under this category

11. Case Report: This category will include interesting neuroimaging data acquired in a small number of patients or voluteers.

The above categories should help us stay ahead of the curve and provide the neuroimaging community a convenient one stop shop for dissemination of data and discussion of novel concepts and technologies. We hope the JNPN will help shape the future of brain research. 